\title{
Maternal betaine status, but not that of choline or methionine, is inversely associated with infant birth weight
}

\author{
Yu-Feng Du ${ }^{1}$, Yuan $\mathrm{Wei}^{2}$, Jing Yang $^{2}$, Zi-Yi Cheng ${ }^{2}$, Xi-Fang Zuo ${ }^{3}$, Tian-Chen Wu ${ }^{1}$, Hui-Feng Shi ${ }^{1}$ and \\ Xiao-Li Wang ${ }^{1 *}$ \\ ${ }^{1}$ Department of Maternal and Child Health, School of Public Health, Peking University, Beijing 100191, People's Republic of \\ China \\ ${ }^{2}$ Obstetrics and Gynecology Department, Peking University Third Hospital, Beijing 100191, People's Republic of China \\ ${ }^{3}$ Obstetrics and Gynecology Department, Tongzhou Maternal and Child Health Hospital of Beijing, Beijing 101101, \\ People's Republic of China \\ (Submitted 7 December 2018 - Final revision received 7 February 2019 - Accepted 26 February 2019 - First published online 7 May 2019)
}

\begin{abstract}
Maternal one-carbon metabolism during pregnancy is crucial for fetal development and programming by DNA methylation. However, evidence on one-carbon biomarkers other than folate is lacking. We, therefore, investigated whether maternal plasma methyl donors, that is, choline, betaine and methionine, are associated with birth outcomes. Blood samples were obtained from 115 women during gestation (median $26 \cdot 3$ weeks, $90 \%$ range 22.7-33.0 weeks). Plasma choline, betaine, methionine and dimethylglycine were measured using HPLC-tandem MS. Multivariate linear and logistic regression models were used to estimate the association between plasma biomarkers and birth weight, birth length, the risk of small-forgestational-age and large-for-gestational-age (LGA). Higher level of maternal betaine was associated with lower birth weight ( $-130 \cdot 3$ (95 \% CI $-244.8,-15.9)$ per $1 \mathrm{SD}$ increment for log-transformed betaine). Higher maternal methionine was associated with lower risk of LGA, and adjusted OR, with $95 \%$ CI for 1 SD increase in methionine concentration was 0.44 (95\% CI 0.21, 0.89). Stratified analyses according to infant sex or maternal plasma homocysteine status showed that reduction in birth weight in relation to maternal betaine was only limited to male infants or to who had higher maternal homocysteine status $(\geq 5 \cdot 1 \mu \mathrm{mol} / \mathrm{l})$. Higher maternal betaine status was associated with reduced birth weight. Maternal methionine was inversely associated with LGA risk. These findings are needed to be replicated in future larger studies.
\end{abstract}

Key words: Pregnancy: Plasma: Betaine: Choline: Methionine: Infants: Birth weight

Higher or lower fetal growth has been implicated in a number of childhood and adult-onset chronic diseases and conditions including type 2 diabetes, hypertension, obesity and CVD ${ }^{(1-6)}$. Epigenetic processes, particularly DNA methylation, regulate fetal programming and development by influencing gene expression $^{(7)}$. Evidence of both animal models and human study has shown that offspring DNA methylation can be modified by maternal intake of methyl nutrients (e.g. folate, choline, betaine and methionine) involved in one-carbon metabolism ${ }^{(8-11)}$. Previous research has extensively studied the potential role of maternal folate intake rather than other methyl donors in influencing fetal growth, and meta-analyses have demonstrated that higher maternal folate intake is associated with an increase in birth weight or with a lower risk of small-forgestational-age (SGA) ${ }^{(12-14)}$.

Similar to folate, choline and its related metabolites also play an important role in one-carbon metabolism. Choline can be irreversibly oxidised to betaine, which further provides a methyl group to homocysteine to form methionine and eventually the universal methyl donor $S$-adenosylmethionine ${ }^{(15)}$. This pathway is paralleled to the 5-methyltetrahydrofolate-dependent route in re-methylation of homocysteine. Choline is also necessary for maintaining the integrity of cell membrane and serves as the precursor for the neurotransmitter acetylcholine ${ }^{(16)}$. Another role of betaine is that it functions as an intracellular osmolyte to protect cells under stress ${ }^{(17)}$. Dimethylglycine (DMG) is formed after betaine providing methyl group to homocysteine. Methionine, also an essential amino acid, is particularly important during fetal development because of high demands for protein synthesis ${ }^{(18)}$. To date, only three epidemiologic studies have explored the association of maternal circulating choline and betaine with birth weight, and the results have been inconsistent $^{(19-21)}$. While a large mother-offspring cohort reported an inverse association between maternal plasma

Abbreviations: DMG, dimethylglycine; GUSTO, Growing Up in Singapore Towards Healthy Outcomes; LGA, large-for-gestational-age; SGA, small-forgestational-age.

* Corresponding author: Professor X.-L. Wang, fax +86 10 62023133, email xlwang@bjmu.edu.cn 
betaine and birth characteristics included, that is, birth weight or birth length or abdominal fat mass ${ }^{(19)}$, other studies reported null association between either biomarkers and birth weight $^{(20,21)}$. Besides, we are aware of only one epidemiological study that has explored amniotic fluid methionine status and offspring birth weight ${ }^{(22)}$; this study showed a positive association. Various factors, such as sample size, differences in gestation age underwent a venipuncture and confounders controlled, could contribute to these inconsistent results.

In this study, we aimed to investigate the association of maternal plasma choline and its related nutrients with birth weight, birth length, SGA and large-for-gestational-age (LGA) by utilising a hospital-based cohort study. Stratified analyses by infant sex were performed because it has been found that restriction of maternal methyl donors intake was associated with offspring's methylation changes of some genes in a sexspecific manner ${ }^{(8)}$. Homocysteine is closely interrelated with methyl donors in one-carbon metabolism, and a causal role for maternal homocysteine in offspring birth weight was observed $^{(23)}$. We thus also evaluated whether the association between assayed biomarkers and birth weight was modified by maternal homocysteine status.

\section{Methods}

\section{Subjects}

A hospital-based cohort study was conducted in Peking University Third Hospital in Beijing, China, between March 2017 and January 2018. Singleton pregnant women aged 18-45 years, conceived naturally and planned to delivery at the participating hospital. Ineligibility was due to an intention to deliver in other hospitals or conceived through assisted reproduction techniques. Eligible women were recruited from the antenatal clinics when they first presented at this hospital. Among 260 eligible women, 176 were willing to participate in our study and agreed to donate a blood sample during the routine blood sampling used for clinical care. Further, sixty-one participants were excluded based on the characteristics of offspring: birth weight lower than $1500 \mathrm{~g}$ ( $n$ 17), early preterm (gestational age $\leq 34$ weeks) ( $n$ 25), had congenital disease ( $n$ 9), malformation $(n 7)$ or chromosomal abnormality ( $n$ 3). Finally, a total of 115 mother-infant pairs were included in the present study (Fig. 1). The study was approved by the Peking University Third Hospital Medical Ethics

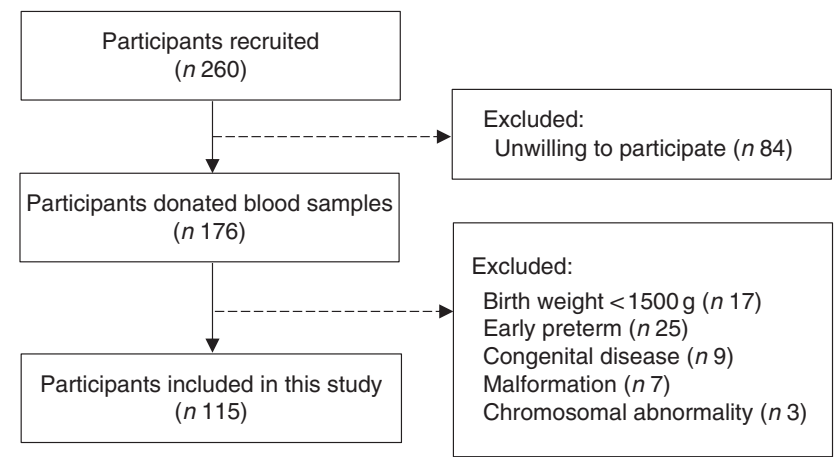

Fig. 1. Flow diagram of participants included in the present study.
Committee (IRB00006761-2016145), and informed consent was obtained from the participants.

\section{Data on main outcomes and covariates}

Information on birth weight and birth length was obtained from the hospital delivery records. Neonates, whose birth weight was $<10$ th percentile or $\geq 90$ th percentile of growth curves based on gestational age and sex ${ }^{(24)}$, were categorised as SGA or LGA, respectively. Gestational age at birth was calculated using the last menstrual period and was subsequently confirmed or revised by early ultrasound. Gestational age based on ultrasound findings was used when there was a discrepancy larger than $10 \mathrm{~d}$. Low birth weight $(n 5)$ and high birth weight $(n 8)$ were not used as interested outcomes due to the small numbers.

Data on maternal characteristics like parity, the age of mother at delivery, medical history, marital status and ethnic group were abstracted from medical records. Weight and height of participants were measured by nurses after admission. Drinking, smoking and weight gain during pregnancy were selfreported by participants at the time of enrolment. Maternal folic acid intake during pregnancy was recalled by mothers after delivery. Pre-pregnancy weight was calculated as the difference between measured weight and self-reported weight gain. Prepregnancy BMI was determined by dividing calculated weight (kg) by height squared $\left(\mathrm{m}^{2}\right)$. Women were categorised into three groups according to smoking: non-smoker, a former smoker and current smoker. Alcohol drinking during pregnancy was also asked (yes or no). Data were separately abstracted by two trained staff, and the consistency was verified.

\section{Sample collection and processing}

Early morning fasting blood samples were collected in EDTAtreated tubes, immediately stored in a $4^{\circ} \mathrm{C}$ refrigerator (storage time was within a maximum of $20 \mathrm{~h}$ ) and were subsequently transported on ice to the laboratory (within $0.5 \mathrm{~h}$ ) for processing. Blood drawing was concurrent with the routine blood sampling. Samples were centrifuged at $3000 \mathrm{rpm}$ for $10 \mathrm{~min}$ at $4^{\circ} \mathrm{C}$, and the aliquoted supernatants were stored at $-80^{\circ} \mathrm{C}$ refrigerator until needed for the analysis.

\section{Measurement of plasma biomarkers}

Concentrations of choline, betaine, DMG, methionine and homocysteine were analysed by HPLC (Shimadzu)-tandem MS (API 4000; MDS Sciex) based on the method of Midttun et al. ${ }^{\text {(25) }}$ with modifications. Briefly, after a $30 \mu \mathrm{l}$ plasma mixed with $5 \mu \mathrm{l}$ internal standard solution followed by a vortex, $150 \mu \mathrm{l}$ of methanol was added to precipitate proteins. The mixture was further vortexed for $30 \mathrm{~s}$ and centrifuged at $13000 \mathrm{~g}$ for $20 \mathrm{~min}$ at $4^{\circ} \mathrm{C}$. A $150 \mu \mathrm{l}$ supernatant was transferred to an Eppendorf tube and $2 \mathrm{~h}$ of freeze-drying was done to remove methanol. Dried samples were then re-dissolved in $150 \mu \mathrm{l}$ of $50 \%$ acetonitrile, and $10 \mu \mathrm{l}$ of supernatant was removed and injected into the HPLC-MS/MS system. Choline- $\mathrm{d}_{9}, \mathrm{DMG}_{-} \mathrm{d}_{6}$ and methionine$\mathrm{d}_{3}$ were used as internal standards. Cysteamine hydrochloride was used as the internal standard for homocysteine. Biomarkers 
of interest were separated by an XBridge BEH Phenyl column $(150 \times 4.6 \mathrm{~mm}, 3.5 \mu \mathrm{m})$ from Waters. Mobile phases consist of phase A $(0 \cdot 1 \%$ formic acid in water) and phase B ( $0 \cdot 1 \%$ formic acid in acetonitrile). We used a column flow rate of $0.5 \mathrm{ml} / \mathrm{min}$, a sample injection volume of $5 \mu \mathrm{l}$ and the column temperature of $40^{\circ} \mathrm{C}$ for the analysis. De-clustering potentials and collision energies were optimised for each biomarker and internal standard. Duplicate control samples were interspersed in each analytical batch to assess laboratory precision. The betweenday CV are as follows: $2.4 \%$ for choline, $6.7 \%$ for betaine, $1.6 \%$ for methionine, $3.4 \%$ for DMG and $1.3 \%$ for homocysteine. All assays were conducted by laboratory technicians, and all of them were blinded to the clinical information of participants.

\section{Statistical analysis}

Biomarker concentrations are not distributed normally and therefore are reported as medians (25th-75th percentiles). Differences in concentrations across groups regarding characteristics about mother and child were examined by the Mann-Whitney $U$ test or Kruskal-Wallis test. For each of the biomarkers, we log transformed and then created a standard deviation score before further analyses. Multiple linear regression models were constructed to assess the effects of maternal biomarkers on continuous outcomes (e.g. birth weight and birth length). The effect size is interpreted as changes in outcomes per $1 \mathrm{SD}$ change in the log-transformed exposure. The association between dichotomous outcomes (SGA and LGA) and maternal blood biomarker was estimated as OR using logistic regression models. We used restricted cubic splines to examine whether non-linear associations between biomarkers with birth weight, birth length, SGA and LGA exist (rms-package in R statistical software), and the result showed that all $P_{\text {non-linearity }}>0 \cdot 1$. In the present study, all participants were married, were nonsmokers and were not drinking during pregnancy. Besides, $96 \cdot 2 \%$ of our participants were Han Chinese. We, thus, did not adjust marriage status, smoking, drinking and ethnicity in multivariate models. Effect values were obtained from models with adjustment for gestational age, gestational age at venipuncture (in 2-week intervals) and fetal sex (model 1), from models additionally controlled for pre-pregnancy BMI $\left(<18.5,18.5-24.9, \geq 25 \mathrm{~kg} / \mathrm{m}^{2}\right)$, maternal age $(<35, \geq 35$ years), parity, gestational diabetes (yes/ no), plasma homocysteine status ( $<90$ percentile, $\geq 90$ percentile) (model 2), as well as from model 3 adjusting for confounders in model 2, plus mutually adjusted four plasma biomarkers. Models for SGA and LGA were not adjusted for newborn's sex and gestational age.

Stratified analyses by infant sex or maternal plasma homocysteine status ( $<75$ percentile, $\geq 75$ percentile) were performed. Covariates adjusted in stratified analyses were selected due to the limited sample size. Covariates that were considered clinically relevant (plasma biomarkers) or with a $P$ value $<0 \cdot 2$ on univariate analysis with response variable were finally included in a multivariate model. To assess the robustness of our results, we performed sensitivity analyses after excluding mothers having gestational diabetes. Comparisons on several key variables (e.g. maternal age, gestational age and birth weight) between study participants and randomly selected 400 women visited this hospital during the study period were performed to check for the representative. In this study, significance was defined as $P<0.05$, and all statistical tests were two tailed. Statistical analyses were conducted by SPSS software (version 24.0) and R statistical software (version 3.4.0).

\section{Results}

As shown in Table 1, the majority of the included mothers were aged $<35$ years $(86 \cdot 1 \%)$, were nulliparous $(82.6 \%)$ and had prepregnancy BMI range from 18.5 to $24.9 \mathrm{~kg} / \mathrm{m}^{2}$ (78.3\%). Notably, $20 \%$ of women suffered gestational diabetes. The newborns, of whom sixty-two were boys (53.9\%), 88.7\% had a birth weight within a normal range (2500-3999 g), and $95.7 \%$ had a gestational age $\geq 37$ weeks. All plasma biomarker concentrations did not differ significantly across groups for each characteristics. Mean for maternal age and pre-pregnancy BMI was 31.5 (SD 3.1 ) years and 21.7 (SD 2.7 ) $\mathrm{kg} / \mathrm{m}^{2}$, respectively.

The association of maternal plasma choline, betaine and DMG and methionine with birth weight and birth length is shown in Table 2. Higher maternal plasma betaine concentrations were associated with lower birth weight in all three adjusted models (model 3: $\beta=-130.3 \mathrm{~g}$ per $1 \mathrm{sD}$ increment; 95\% CI $-244 \cdot 8$, $15.9 \mathrm{~g}$ ). A significant inverse association was also seen for choline with birth weight in model 1 , but this association weakened sharply and became not statistically significant after inclusion of plasma betaine, DMG and methionine in model 3. None of the biomarkers was associated with birth length in our study.

Table 3 shows the association of maternal plasma biomarkers with SGA and LGA risk. A strong inverse relationship between maternal methionine status and LGA risk was observed in either crude or adjusted models. The adjusted OR was 0.44 (95\% CI $0 \cdot 21,0 \cdot 89)$ for every $1 \mathrm{sD}$ increment of methionine concentrations. Plasma choline and betaine was also inversely associated with LGA risk and crude OR was 0.51 (95\% CI 0.27, 0.96) and $0 \cdot 48$ (95\% CI 0.26, 0.88), respectively, but these associations did not survived after further controlling for covariates. Of note, both maternal betaine and methionine status were not associated with the increased risk of SGA.

Stratified analysis based on infant sex showed that reduction in birth weight related to maternal betaine status only limited to male infants $(\beta$ : $-156.0 \mathrm{~g}$ per $1 \mathrm{SD}$ increment, $95 \% \mathrm{CI}-305 \cdot 1$, 6.9; $P=0.041$ ) (Table 4). Besides, the inverse association between maternal betaine status with birth weight were mainly significant among women with higher homocysteine status $(\geq 5 \cdot 1 \mu \mathrm{mol} / \mathrm{l})(\beta:-206 \cdot 3 \mathrm{~g}$ per $1 \mathrm{sD}$ increment, 95\% CI $-402 \cdot 5$, $-10 \cdot 0 ; P=0 \cdot 040$ ) (Table 5). The participants in the present study did not significantly differ from randomly selected 400 women concerning key variables as follows: maternal age $(P=0.966)$, gestational age $(P=0 \cdot 164)$, ethnicity $(P=0 \cdot 255)$, gestational diabetes $(P=0 \cdot 127)$ and birth weight $(P=0.455)$. Sensitivity analyses for main results yielded similar results, but the association was slightly weaker (data not shown).

\section{Discussion}

To our knowledge, this is the first study to explore the role of maternal choline and its related metabolites in one-carbon 
Table 1. Maternal biomarker concentrations ( $\mu \mathrm{mol} / \mathrm{l}$ ) according to maternal and infant characteristics* (Numbers and percentages; medians and 25th, 75th percentiles)

\begin{tabular}{|c|c|c|c|c|c|c|c|c|c|c|}
\hline & \multirow[b]{2}{*}{$n$} & \multirow[b]{2}{*}{$\%$} & \multicolumn{2}{|c|}{ Choline } & \multicolumn{2}{|c|}{ Betaine } & \multicolumn{2}{|c|}{ Dimethylglycine } & \multicolumn{2}{|c|}{ Methionine } \\
\hline & & & Median & $\begin{array}{l}\text { 25th, } 75 \text { th } \\
\text { percentile }\end{array}$ & Median & $\begin{array}{l}\text { 25th, 75th } \\
\text { percentile }\end{array}$ & Median & $\begin{array}{l}\text { 25th, 75th } \\
\text { percentile }\end{array}$ & Median & $\begin{array}{l}\text { 25th, } 75 \text { th } \\
\text { percentile }\end{array}$ \\
\hline \multicolumn{11}{|l|}{ Age (years) } \\
\hline$<35$ & 99 & $86 \cdot 1$ & $9 \cdot 1$ & $7 \cdot 0,10 \cdot 6$ & $15 \cdot 1$ & $12 \cdot 3,17 \cdot 3$ & $1 \cdot 6$ & $1.3,1.9$ & $18 \cdot 7$ & $15 \cdot 7,21 \cdot 3$ \\
\hline$\geq 35$ & 16 & $13 \cdot 9$ & 8.4 & $7 \cdot 8,10 \cdot 4$ & 14.5 & $11 \cdot 1,16 \cdot 3$ & 1.6 & $1 \cdot 3,1 \cdot 8$ & $18 \cdot 8$ & $16 \cdot 1,22 \cdot 9$ \\
\hline$P$ & & & \multicolumn{2}{|c|}{0.897} & \multicolumn{2}{|c|}{0.482} & \multicolumn{2}{|c|}{0.994} & \multirow{2}{*}{\multicolumn{2}{|c|}{0.616}} \\
\hline \multicolumn{10}{|c|}{ Pre-pregnancy BMI $\left(\mathrm{kg} / \mathrm{m}^{2}\right)$} & \\
\hline$<18.5$ & 13 & $11 \cdot 3$ & 8.9 & $7 \cdot 0,9 \cdot 4$ & $15 \cdot 7$ & $12 \cdot 7,17 \cdot 4$ & 1.5 & $1 \cdot 3,1 \cdot 7$ & $17 \cdot 1$ & $13 \cdot 3,19 \cdot 5$ \\
\hline $18.5-24.9$ & 90 & $78 \cdot 3$ & 8.9 & $7 \cdot 1,10 \cdot 6$ & 14.9 & $12 \cdot 2,17 \cdot 0$ & $1 \cdot 6$ & $1.4,1.9$ & $18 \cdot 8$ & $15 \cdot 7,21 \cdot 3$ \\
\hline$\geq 25$ & 12 & $10 \cdot 4$ & $10 \cdot 2$ & $7 \cdot 8,11.5$ & $16 \cdot 5$ & $11 \cdot 3,18 \cdot 1$ & 1.5 & $1 \cdot 3,1 \cdot 7$ & $20 \cdot 0$ & $17 \cdot 6,23 \cdot 5$ \\
\hline$P$ & & & \multicolumn{2}{|c|}{0.484} & \multicolumn{2}{|c|}{0.874} & \multicolumn{2}{|c|}{0.435} & \multicolumn{2}{|c|}{0.195} \\
\hline \multicolumn{11}{|l|}{ Parity } \\
\hline Nulliparous & 95 & $82 \cdot 6$ & 8.9 & $7 \cdot 0,10 \cdot 7$ & $15 \cdot 3$ & $12 \cdot 3,18 \cdot 2$ & 1.6 & $1.3,1.9$ & $18 \cdot 8$ & $15 \cdot 7,21 \cdot 5$ \\
\hline Multiparous & 20 & $17 \cdot 4$ & $9 \cdot 2$ & $7 \cdot 7,10 \cdot 0$ & $14 \cdot 0$ & $11.9,16.5$ & $1 \cdot 6$ & $1.4,1.7$ & 18.5 & $15 \cdot 5,19 \cdot 7$ \\
\hline$P$ & & & \multicolumn{2}{|c|}{0.831} & \multicolumn{2}{|c|}{0.341} & \multicolumn{2}{|c|}{0.825} & \multicolumn{2}{|c|}{0.545} \\
\hline \multicolumn{11}{|c|}{ Gestational diabetes } \\
\hline No & 92 & $80 \cdot 0$ & $9 \cdot 3$ & $7 \cdot 1,10 \cdot 6$ & $15 \cdot 4$ & $12 \cdot 8,17 \cdot 4$ & 1.6 & $1 \cdot 3,1 \cdot 9$ & $19 \cdot 1$ & $15 \cdot 8,21 \cdot 6$ \\
\hline Yes & 23 & $20 \cdot 0$ & $8 \cdot 1$ & $7 \cdot 0,9 \cdot 2$ & $14 \cdot 8$ & $11 \cdot 2,16 \cdot 3$ & 1.5 & $1 \cdot 3,1 \cdot 8$ & $17 \cdot 0$ & $15 \cdot 2,20 \cdot 3$ \\
\hline$P$ & & & \multicolumn{2}{|c|}{0.177} & \multicolumn{2}{|c|}{$0 \cdot 158$} & \multicolumn{2}{|c|}{0.534} & \multicolumn{2}{|c|}{0.206} \\
\hline \multicolumn{11}{|l|}{ Fetal sex } \\
\hline Male & 62 & 53.9 & $8 \cdot 8$ & $7 \cdot 3,11 \cdot 2$ & $14 \cdot 7$ & $11 \cdot 9,16 \cdot 8$ & 1.5 & $1 \cdot 3,1.9$ & $18 \cdot 0$ & $15 \cdot 7,20 \cdot 7$ \\
\hline Female & 53 & $46 \cdot 1$ & $9 \cdot 1$ & $7 \cdot 1,9 \cdot 9$ & $15 \cdot 7$ & $13 \cdot 5,18 \cdot 2$ & 1.6 & $1 \cdot 5,1 \cdot 8$ & $19 \cdot 3$ & $16 \cdot 0,21 \cdot 5$ \\
\hline$P$ & & & \multicolumn{2}{|c|}{0.419} & & & & 193 & & 245 \\
\hline Gestational ag & & & & & & & & & & \\
\hline$<37$ & 5 & $4 \cdot 3$ & $9 \cdot 3$ & $9 \cdot 2,9 \cdot 8$ & $14 \cdot 2$ & $13 \cdot 4,15 \cdot 7$ & 1.6 & $1.5,1 \cdot 7$ & $15 \cdot 2$ & $14 \cdot 8,15 \cdot 3$ \\
\hline$\geq 37$ & 110 & $95 \cdot 7$ & 8.9 & $7 \cdot 0,10 \cdot 5$ & $15 \cdot 1$ & $12 \cdot 1,17 \cdot 3$ & 1.6 & $1 \cdot 3,1 \cdot 8$ & $18 \cdot 9$ & $16 \cdot 0,21 \cdot 5$ \\
\hline$P$ & & & & 546 & & & & 967 & & 068 \\
\hline Birth weight ( $\mathrm{g}$ & & & & & & & & & & \\
\hline$<2500$ & 5 & $4 \cdot 3$ & 9.8 & $9 \cdot 8,10 \cdot 1$ & $17 \cdot 1$ & $14.9,18.9$ & 1.5 & $1 \cdot 2,1 \cdot 7$ & $17 \cdot 0$ & $16 \cdot 7,19 \cdot 3$ \\
\hline 2500-3999 & 102 & 88.7 & 8.9 & $7 \cdot 0,10 \cdot 7$ & $15 \cdot 2$ & $12 \cdot 2,17 \cdot 3$ & 1.6 & $1 \cdot 3,1 \cdot 8$ & $18 \cdot 8$ & $15 \cdot 7,21 \cdot 4$ \\
\hline$\geq 4000$ & 8 & $7 \cdot 0$ & 8.0 & $7 \cdot 0,8 \cdot 8$ & $13 \cdot 5$ & $11 \cdot 3,15 \cdot 5$ & 1.5 & $1 \cdot 3,2 \cdot 1$ & $17 \cdot 9$ & $16 \cdot 1,21 \cdot 8$ \\
\hline$P$ & & & & 249 & & 73 & & 840 & & 952 \\
\hline
\end{tabular}

${ }^{*} P$ values are obtained from the Mann-Whitney $U$ test or Kruskal-Wallis test.

Table 2. Associations of maternal biomarker concentrations ( $\mu \mathrm{mol} / \mathrm{l})$ with birth weight and birth length* ( $\beta$-Coefficients and $95 \%$ confidence intervals)

\begin{tabular}{|c|c|c|c|c|c|c|}
\hline & \multicolumn{3}{|c|}{ Birth weight $(\mathrm{g})$} & \multicolumn{3}{|c|}{ Birth length $(\mathrm{cm})$} \\
\hline & $\beta$ & $95 \% \mathrm{Cl}$ & $P$ & $\beta$ & $95 \% \mathrm{Cl}$ & $P$ \\
\hline \multicolumn{7}{|l|}{ Choline } \\
\hline Model 1 & $-91 \cdot 8$ & $-174 \cdot 8,-8 \cdot 7$ & 0.031 & -0.31 & $-0.64,0.01$ & 0.055 \\
\hline Model 2 & -73.5 & $-159 \cdot 3,12 \cdot 4$ & 0.092 & -0.30 & $-0.64,0.03$ & 0.078 \\
\hline Model 3 & $-37 \cdot 6$ & $-149 \cdot 9,74 \cdot 7$ & 0.508 & -0.25 & $-0.71,0.20$ & 0.266 \\
\hline \multicolumn{7}{|l|}{ Betaine } \\
\hline Model 1 & $-115 \cdot 4$ & $-195 \cdot 3,-35 \cdot 5$ & 0.005 & -0.26 & $-0.57,0.06$ & 0.110 \\
\hline Model 2 & $-104 \cdot 1$ & $-186 \cdot 2,-22 \cdot 0$ & 0.013 & -0.23 & $-0.56,0.10$ & 0.166 \\
\hline Model 3 & $-130 \cdot 3$ & $-244 \cdot 8,-15 \cdot 9$ & 0.026 & -0.11 & $-0.58,0.35$ & 0.623 \\
\hline \multicolumn{7}{|c|}{ Dimethylglycine } \\
\hline Model 1 & -36.9 & $-120 \cdot 1,46 \cdot 3$ & 0.381 & -0.14 & $-0.46,0.18$ & 0.405 \\
\hline Model 2 & $-22 \cdot 6$ & $-108 \cdot 7,63 \cdot 5$ & 0.604 & -0.16 & $-0.50,0.18$ & 0.353 \\
\hline Model 3 & 79.4 & $-35 \cdot 1,193.9$ & 0.172 & 0.08 & $-0.38,0.54$ & 0.721 \\
\hline \multicolumn{7}{|l|}{ Methionine } \\
\hline Model 1 & -21.5 & $-106 \cdot 1,63 \cdot 2$ & 0.616 & -0.19 & $-0.51,0.13$ & 0.246 \\
\hline Model 2 & $-7 \cdot 8$ & $-93.9,78.4$ & 0.858 & $-0 \cdot 15$ & $-0.49,0.19$ & 0.378 \\
\hline Model 3 & $2 \cdot 0$ & $-87 \cdot 0,90 \cdot 9$ & 0.965 & -0.09 & $-0.45,0.27$ & 0.613 \\
\hline
\end{tabular}

metabolism in infant growth in a Chinese population. This study shows that high maternal plasma betaine concentrations were associated with the lower birth weight of infants. This association seemed to be stronger in male infants, or those had higher maternal homocysteine status. Higher maternal plasma methionine was associated with lower risk of LGA. 
Table 3. Risks of small-for-gestational-age (SGA)/large-for-gestational-age (LGA) according to each maternal biomarker concentrations $(\mu \mathrm{mol} /)^{*}$

(Odds ratios and $95 \%$ confidence intervals)

\begin{tabular}{|c|c|c|c|c|c|c|}
\hline & \multicolumn{3}{|c|}{ SGA } & \multicolumn{3}{|c|}{ LGA } \\
\hline & OR & $95 \% \mathrm{Cl}$ & $P$ & OR & $95 \% \mathrm{Cl}$ & $P$ \\
\hline \multicolumn{7}{|l|}{ Choline } \\
\hline Model 1 & 1.48 & $0.85,2.59$ & 0.170 & 0.51 & $0.27,0.96$ & 0.036 \\
\hline Model 2 & 1.32 & $0 \cdot 68,2 \cdot 56$ & 0.416 & 0.55 & $0.28,1.09$ & 0.086 \\
\hline Model 3 & 3.41 & $0.78,14.80$ & 0.102 & 0.99 & $0.37,2.63$ & 0.987 \\
\hline \multicolumn{7}{|l|}{ Betaine } \\
\hline Model 1 & 1.20 & $0.65,2.25$ & 0.560 & 0.48 & $0.26,0.88$ & 0.017 \\
\hline Model 2 & 0.97 & $0.50,1.88$ & 0.928 & 0.45 & $0.22,0.92$ & 0.028 \\
\hline Model 3 & 0.73 & $0.24,2 \cdot 21$ & 0.574 & 0.49 & $0.16,1.50$ & 0.211 \\
\hline \multicolumn{7}{|c|}{ Dimethylglycine } \\
\hline Model 1 & 0.60 & $0.28,1.27$ & 0.181 & 0.73 & $0.41,1.29$ & 0.405 \\
\hline Model 2 & 0.94 & $0.49,1.80$ & 0.859 & 0.51 & $0.24,1.09$ & 0.083 \\
\hline Model 3 & 0.55 & $0.16,1.84$ & 0.329 & $1 \cdot 13$ & $0.37,3.42$ & 0.828 \\
\hline \multicolumn{7}{|l|}{ Methionine } \\
\hline Model 1 & 0.61 & $0.31,1 \cdot 22$ & 0.163 & 0.46 & $0.25,0.83$ & 0.010 \\
\hline Model 2 & 0.59 & $0.24,1.42$ & 0.236 & 0.41 & $0.21,0.79$ & 0.007 \\
\hline Model 3 & 0.51 & $0.19,1.40$ & 0.193 & 0.44 & $0.21,0.89$ & 0.023 \\
\hline
\end{tabular}

* Model 1: crude models. Model 2: adjusted for gestational age at venipuncture, gestational diabetes, maternal age, pre-pregnancy BM $\left(<18.5,18.5-24.9, \geq 25 \mathrm{~kg} / \mathrm{m}^{2}\right)$, parity, plasma homocysteine status ( $<90$ percentile, $\geq 90$ percentile). Model 3: covariates in model 2 and mutually adjusted for plasma choline, betaine, dimethylglycine and methionine.

Table 4. Association between maternal biomarker concentrations $(\mu \mathrm{mol} / \mathrm{l})$ with birth weight according to infant sex ${ }^{\star}$ ( $\beta$-Coefficients and $95 \%$ confidence intervals)

\begin{tabular}{|c|c|c|c|c|c|c|}
\hline & \multicolumn{3}{|c|}{ Male $(n 62)$} & \multicolumn{3}{|c|}{ Female $(n 53)$} \\
\hline & Adjusted $\beta$ & $95 \% \mathrm{Cl}$ & $P$ & Adjusted $\beta$ & $95 \% \mathrm{Cl}$ & $P$ \\
\hline Choline & $-25 \cdot 4$ & $-173 \cdot 6,122 \cdot 8$ & 0.733 & $-60 \cdot 2$ & $-233 \cdot 7,113.4$ & 0.489 \\
\hline Betaine & $-156 \cdot 0$ & $-305 \cdot 1,-6 \cdot 9$ & 0.041 & -86.5 & $-258 \cdot 8,85 \cdot 8$ & 0.318 \\
\hline Dimethylglycine & $48 \cdot 4$ & $-82 \cdot 6,179 \cdot 5$ & 0.462 & $90 \cdot 6$ & $-98 \cdot 5,279.8$ & 0.340 \\
\hline Methionine & $100 \cdot 7$ & $-13 \cdot 4,214 \cdot 8$ & 0.082 & $-64 \cdot 0$ & $-197 \cdot 7,69 \cdot 7$ & 0.341 \\
\hline
\end{tabular}

* Adjusted for gestational age, gestational diabetes and mutually adjusted for plasma choline, betaine, dimethylglycine and methionine.

Table 5. Association between maternal biomarker concentrations ( $\mu \mathrm{mol} / \mathrm{l}$ ) with birth weight according to maternal plasma homocysteine status*

( $\beta$-Coefficients and $95 \%$ confidence intervals)

\begin{tabular}{|c|c|c|c|c|c|c|}
\hline & \multicolumn{3}{|c|}{ Homocysteine $(<5.1 \mu \mathrm{mol} / \mathrm{l})$} & \multicolumn{3}{|c|}{ Homocysteine $(\geq 5.1 \mu \mathrm{mol} / \mathrm{l})$} \\
\hline & Adjusted $\beta \dagger$ & $95 \% \mathrm{Cl}$ & $P$ & Adjusted $\beta \dagger$ & $95 \% \mathrm{Cl}$ & $P$ \\
\hline Choline & $-45 \cdot 0$ & $-182.4,92 \cdot 3$ & 0.516 & $-52 \cdot 6$ & $-225 \cdot 4,120 \cdot 1$ & 0.532 \\
\hline Betaine & -48.6 & $-196 \cdot 5,99 \cdot 4$ & 0.516 & $-206 \cdot 3$ & $-402 \cdot 5,-10 \cdot 0$ & 0.040 \\
\hline Dimethylglycine & 59.9 & $-88 \cdot 3,208 \cdot 0$ & 0.423 & 62.3 & $-118 \cdot 5,243 \cdot 2$ & 0.480 \\
\hline Methionine & 3.7 & $-99 \cdot 7,107 \cdot 1$ & 0.943 & $22 \cdot 1$ & $-161 \cdot 0,205 \cdot 1$ & 0.804 \\
\hline
\end{tabular}

* Stratification is based on 75th percentile of maternal homocysteine concentrations in this study.

† Adjusted for gestational age, infant sex, gestational age at venipuncture (in 2-week intervals), gestational diabetes, and mutually adjusted for plasma choline, betaine, dimethylglycine and methionine.

Our finding that maternal plasma betaine concentrations were negatively associated with birth weight is strengthened by results from Growing Up in Singapore Towards Healthy Outcomes (GUSTO) cohort study ${ }^{(19)}$, the largest mother-offspring cohort to date explored such an association. The GUSTO study also showed that higher maternal plasma betaine was associated with less abdominal fat mass, shorter birth length and increased risk of $\mathrm{SGA}^{(19)}$. However, another study conducted in the Netherlands found a null association, while an inverse association was observed for umbilical cords betaine status with birth weight ${ }^{(21)}$. Both the GUSTO study and our study collected fasting blood samples. The difference in study population might partly explain these inconsistent findings because the dietary pattern has been found to have a profound effect on methyl-donor biomarker status ${ }^{(26)}$. The significant positive association between maternal betaine status and SGA risk, showed in GUSTO, was not found in our study; this can be explained by the limited power of the analysis resulted from the small number of SGA infants. Besides, a recent study showed that maternal betaine supplementation prevented fetal and 
placental overgrowth in high-fat-fed mice ${ }^{(27)}$. Maternal plasma betaine have been found to remain constant from twenty-two gestational weeks to the rest of pregnancy ${ }^{(28,29)}$, a stage covering the period of our blood sample collection. A strong correlation between maternal betaine status and cord blood betaine status was observed ${ }^{(30)}$.

Evidence from animal and human adult betaine supplementation trials as well as the GUSTO study suggested that the possible mechanisms of the observed decrease in birth weight related to betaine may involve adiposity reductions in infants $^{(19,31,32)}$. Betaine supplementation has been shown to promote muscular fatty acid uptake and oxidation via upregulation of related genes in pigs ${ }^{(33)}$. Increased betainedependent remethylation of homocysteine may reduce the availability of acetyl-coenzyme A, a substrate required for fatty acid synthesis and fat deposition ${ }^{(34)}$. Betaine also promotes the biosynthesis of carnitine by providing a methyl group. Carnitine is necessary for the oxidation of long-chain fatty acids and has been associated with reduced carcass fat content in pigs ${ }^{(31)}$

In the stratified analyses, we found that significant association between betaine and birth weight was mainly among male infants. In animal models, epigenetic changes and phenotypic differences in the offspring caused by restriction of periconceptional methyl donors intake were most pronounced in males ${ }^{(8)}$. Previous studies conducted among adults also demonstrated that favourable body composition (e.g. lower weight and lower body fat) associated with higher serum betaine was mainly seen for male participants ${ }^{(35,36)}$. This sexual dimorphism might involve the differential gene methylation alterations in response to betaine between males and females. It was observed that higher maternal intake of betaine is associated with lower cord blood methylation in males only ${ }^{(10)}$. A randomised controlled trial of peri-conceptional nutrient supplementation, which used supplements containing nutrients involved in one-carbon metabolism such as folic acid and vitamin $\mathrm{B}_{12}$, showed little overlap between males and females in the gene that underwent methylation alterations associated with supplementation ${ }^{(37)}$.

We noted a substantial reduction in birth weight related to the higher maternal betaine and homocysteine concentrations. Maternal betaine was inversely associated with homocysteine only when folate status was low ${ }^{(29,38)}$. However, a small but significant positive association between betaine and homocysteine was observed in our study (Spearman correlation coefficient was $0 \cdot 20, P=0.03$ ). We speculated that folate status, $\mathrm{B}$ vitamins and genetic variants might explain the inconsistent correlation. We assumed that our participants were in a relatively folate-replete condition because $98,89,60,24 \%$ of our participants reported that they took folic acid before pregnancy, during the first, the second and third trimester of pregnancy, respectively. As for the adherence, $84 \%$ of mothers reported that they took folic acid in $\geq 8$ out of $10 \mathrm{~d}$. In addition, some other important predictors of homocysteine status such as methylenetetrahydrofolate reductase $677 \mathrm{C} \rightarrow \mathrm{T}$ polymorphism and $\mathrm{B}$ vitamins $\left(\mathrm{B}_{2}, \mathrm{~B}_{6}\right.$ and $\left.\mathrm{B}_{12}\right)$, all of which were not determined in our study, modulate the homocysteine-betaine relationship ${ }^{(38)}$. The effect of betaine and homocysteine on birth weight cannot be differentiated because of their correlation, and our observed birth weight reduction might reflect the effect of both biomarkers. The inverse association between homocysteine and birth weight has been well illustrated in previous studies ${ }^{(23,39)}$, and the mechanisms involve the impaired placental perfusion and function, resulting from the damaging effect on endothelial cell $^{(40)}$. Further studies are needed to clarify the biological mechanisms of these results. Given the limited sample size, results based on stratified analyses might be a chance finding.

The demand for choline is high during pregnancy due to the accelerated one-carbon metabolism and the formation of new membranes during cellular division ${ }^{(41)}$. However, previous studies $^{(20,21)}$ together with ours failed to find association between maternal choline and birth weight. It has been shown that plasma choline increased progressively from fifteen gestational weeks throughout the rest of pregnancy ${ }^{(29)}$, mainly due to the mobilisation of maternal hepatic choline stores and endogenous synthesis enhanced by oestrogen ${ }^{(42)}$. Associations between choline status with outcomes might be masked because we collected blood samples in a pregnancy stage during which plasma choline is increasing. Furthermore, it should also be noted that maternal plasma choline at delivery was not associated with cord plasma choline ${ }^{(30)}$.

Although maternal methionine was unrelated to birth weight, a $56 \%$ decreased risk of LGA per $1 \mathrm{sD}$ increment of maternal plasma methionine was observed in our study. LGA is related to prolonged delivery, excessive maternal haemorrhage and caesarean sections ${ }^{(43)}$, and an increased risk of obesity, diabetes and CVD later in life ${ }^{(5,6)}$. Recently a study in sows has demonstrated that methionine supplementation during late gestation lowered the TAG levels ${ }^{(44)}$. Furthermore, the thirdtrimester maternal TAG status has been considered as an independent risk predictor of macrosomia ${ }^{(45,46)}$. It is possible that TAG status might help in interpreting this result. This interpretation is highly putative due to the limited evidence. This intriguing finding warrants further confirmation.

Key strengths of the present study are our direct measurements of methyl donor nutrition and its longitudinal design. Several limitations of our study should also be noted. First, the small number of participants limits the power of our study. Second, maternal mental health conditions that are considered to have an effect on infant growth ${ }^{(47)}$ were unfortunately not assessed in our study. Third, a single measurement may not directly reflect the methyl donor nutrition during the whole pregnancy because of the variations in concentrations of some plasma one-carbon biomarkers such as choline and DMG throughout gestation ${ }^{(29)}$. Blood sample collection during different stages of pregnancy from each study subject should be considered in future studies. Moreover, recruitment conducted in a tertiary referral hospital reduced the external validity of our results. However, our study was restricted to an assumingly healthy population, and thus findings can be extrapolated to the general population to some extent. Of note, $70 \%$ of participants in our study were white-collar workers, representing a relatively high socio-economic status of our participants.

In conclusion, our findings showed that maternal plasma betaine showed a strong inverse relationship to birth weight. Maternal methionine status showed a protective effect on LGA risk. Maternal betaine and methionine may have a role in 
preventing fetal overgrowth. Given the implications of inappropriate fetal growth for later-life chronic disease risk, more investigations exploring the effect of maternal one-carbon biomarkers on fetal growth are warranted. Our results require further validation in larger mother-infant cohorts.

\section{Acknowledgements}

We gratefully acknowledge the cooperation of the study participants.

This work is supported by the National Key Research and Development Program of Reproductive Health and Major Birth Defects Control and Prevention (2016YFC1000400).

Y.-F. D. performed the statistical analyses and interpreted the results and wrote the paper; Y. W., J. Y. and Z.-Y. C. participated in the participant's recruitment, data collection, blood sample collection and study design; Y.-F. D. and X.-F. Z. abstracted data from medical records; T.-C. W. and H.-F. S. offered help in the assay of biomarkers. X.-L. W. designed the research, supervised and contributed to manuscript writing. All authors revised the manuscript and read and approved the final manuscript.

The authors declare that they have no conflicts of interest

\section{References}

1. Whincup PH, Kaye SJ, Owen CG, et al. (2008) Birth weight and risk of type 2 diabetes: a systematic review. JAMA 300, 2886-2897.

2. Yu ZB, Han SP, Zhu GZ, et al. (2011) Birth weight and subsequent risk of obesity: a systematic review and meta-analysis. Obes Rev 12, 525-542.

3. McNamara BJ, Gubhaju L, Chamberlain C, et al. (2012) Early life influences on cardio-metabolic disease risk in aboriginal populations-what is the evidence? A systematic review of longitudinal and case-control studies. Int J Epidemiol 41, 1661-1682.

4. Zarrati M, Shidfar F, Razmpoosh E, et al. (2013) Does low birth weight predict hypertension and obesity in schoolchildren? Ann Nutr Metab 63, 69-76.

5. Taal HR, Vd Heijden AJ, Steegers EA, et al. (2013) Small and large size for gestational age at birth, infant growth, and childhood overweight. Obesity (Silver Spring) 21, 1261-1268.

6. Boney CM, Verma A, Tucker R, et al. (2005) Metabolic syndrome in childhood: association with birth weight, maternal obesity, and gestational diabetes mellitus. Pediatrics 115 , e290-e296.

7. Smith ZD \& Meissner A (2013) DNA methylation: roles in mammalian development. Nat Rev Genet 14, 204-220.

8. Sinclair KD, Allegrucci C, Singh R, et al. (2007) DNA methylation, insulin resistance, and blood pressure in offspring determined by maternal periconceptional $B$ vitamin and methionine status. Proc Natl Acad Sci U S A 104, 19351-19356.

9. Waterland RA, Dolinoy DC, Lin JR, et al. (2006) Maternal methyl supplements increase offspring DNA methylation at Axin Fused. Genesis 44, 401-406.

10. Boeke CE, Baccarelli A, Kleinman KP, et al. (2012) Gestational intake of methyl donors and global LINE-1 DNA methylation in maternal and cord blood: prospective results from a folatereplete population. Epigenetics 7, 253-260.

11. Pauwels S, Ghosh M, Duca RC, et al. (2017) Maternal intake of methyl-group donors affects DNA methylation of metabolic genes in infants. Clin Epigenetics 9, 16.
12. Hodgetts VA, Morris RK, Francis A, et al. (2015) Effectiveness of folic acid supplementation in pregnancy on reducing the risk of small-for-gestational age neonates: a population study, systematic review and meta-analysis. $B J O G \mathbf{1 2 2}$, 478-490.

13. Fekete K, Berti C, Trovato M, et al. (2012) Effect of folate intake on health outcomes in pregnancy: a systematic review and meta-analysis on birth weight, placental weight and length of gestation. Nutr J 11, 75 .

14. Zhang Q, Wang Y, Xin X, et al. (2017) Effect of folic acid supplementation on preterm delivery and small for gestational age births: a systematic review and meta-analysis. Reprod Toxicol 67, 35-41.

15. Ueland PM (2011) Choline and betaine in health and disease. J Inherit Metab Dis 34, 3-15.

16. Zeisel SH \& da Costa KA (2009) Choline: an essential nutrient for public health. Nutr Rev 67, 615-623.

17. Ueland PM, Holm PI \& Hustad S (2005) Betaine: a key modulator of one-carbon metabolism and homocysteine status. Clin Chem Lab Med 43, 1069-1075.

18. Rees WD, Wilson FA \& Maloney CA (2006) Sulfur amino acid metabolism in pregnancy: the impact of methionine in the maternal diet. J Nutr 136, 1701s-1705s.

19. van Lee L, Tint MT, Aris IM, et al. (2016) Prospective associations of maternal betaine status with offspring weight and body composition at birth: the Growing Up in Singapore Towards Healthy Outcomes (GUSTO) cohort study. Am J Clin Nutr 104, 1327-1333.

20. Ivorra C, Garcia-Vicent C, Chaves FJ, et al. (2012) Metabolomic profiling in blood from umbilical cords of low birth weight newborns. J Transl Med 10, 142.

21. Hogeveen M, den Heijer M, Semmekrot BA, et al. (2013) Umbilical choline and related methylamines betaine and dimethylglycine in relation to birth weight. Pediatr Res $\mathbf{7 3}$, 783-787.

22. Monsen AL, Schneede J \& Ueland PM (2006) Mid-trimester amniotic fluid methionine concentrations: a predictor of birth weight and length. Metabolism 55, 1186-1191.

23. Yajnik CS, Chandak GR, Joglekar C, et al. (2014) Maternal homocysteine in pregnancy and offspring birthweight: epidemiological associations and Mendelian randomization analysis. Int J Epidemiol 43, 1487-1497.

24. Mikolajczyk RT, Zhang J, Betran AP, et al. (2011) A global reference for fetal-weight and birthweight percentiles. Lancet 377, 1855-1861.

25. Midttun O, Kvalheim G \& Ueland PM (2013) High-throughput, low-volume, multianalyte quantification of plasma metabolites related to one-carbon metabolism using HPLC-MS/MS. Anal Bioanal Chem 405, 2009-2017.

26. Dominguez-Salas P, Moore SE, Cole D, et al. (2013) DNA methylation potential: dietary intake and blood concentrations of one-carbon metabolites and cofactors in rural African women. Am J Clin Nutr 97, 1217-1227.

27. Joselit Y, Nanobashvili K, Jack-Roberts C, et al. (2018) Maternal betaine supplementation affects fetal growth and lipid metabolism of high-fat fed mice in a temporalspecific manner. Nutr Diabetes $\mathbf{8}, 41$.

28. Velzing-Aarts FV, Holm PI, Fokkema MR, et al. (2005) Plasma choline and betaine and their relation to plasma homocysteine in normal pregnancy. Am J Clin Nutr 81, 1383-1389.

29. Fernandez-Roig S, Cavalle-Busquets P, Fernandez-Ballart JD, et al. (2013) Low folate status enhances pregnancy changes in plasma betaine and dimethylglycine concentrations and the association between betaine and homocysteine. Am J Clin Nutr 97, 1252-1259. 
30. Visentin CE, Masih S, Plumptre L, et al. (2015) Maternal choline status, but not fetal genotype, influences cord plasma choline metabolite concentrations. J Nutr 145, 1491-1497.

31. Eklund M, Bauer E, Wamatu J, et al. (2005) Potential nutritional and physiological functions of betaine in livestock. Nutr Res Rev 18, 31-48.

32. Cholewa JM, Guimaraes-Ferreira L \& Zanchi NE (2014) Effects of betaine on performance and body composition: a review of recent findings and potential mechanisms. Amino Acids $\mathbf{4 6}$, $1785-1793$.

33. Li S, Wang H, Wang X, et al. (2017) Betaine affects muscle lipid metabolism via regulating the fatty acid uptake and oxidation in finishing pig. J Anim Sci Biotechnol 8, 72.

34. Lawrence BV, Schinckel AP, Adeola O, et al. (2002) Impact of betaine on pig finishing performance and carcass composition. J Anim Sci 80, 475-482.

35. Gao X, Randell E, Zhou $\mathrm{H}$, et al. (2018) Higher serum choline and betaine levels are associated with better body composition in male but not female population. PLOS ONE 13, e0193114.

36. Chen YM, Liu Y, Liu YH, et al. (2015) Higher serum concentrations of betaine rather than choline is associated with better profiles of DXA-derived body fat and fat distribution in Chinese adults. Int J Obes 39, 465-471.

37. Khulan B, Cooper WN, Skinner BM, et al. (2012) Periconceptional maternal micronutrient supplementation is associated with widespread gender related changes in the epigenome: a study of a unique resource in the Gambia. Hum Mol Genet 21, 2086-2101.

38. Holm PI, Hustad S, Ueland PM, et al. (2007) Modulation of the homocysteine-betaine relationship by methylenetetrahydrofolate reductase $677 \mathrm{C} \rightarrow \mathrm{t}$ genotypes and B-vitamin status in a large-scale epidemiological study. J Clin Endocrinol Metab 92, 1535-1541.

39. Hogeveen M, Blom HJ \& den Heijer M (2012) Maternal homocysteine and small-for-gestational-age offspring: systematic review and meta-analysis. Am J Clin Nutr 95, 130-136.

40. Gaiday AN, Tussupkaliyev AB, Bermagambetova SK, et al. (2018) Effect of homocysteine on pregnancy: a systematic review. Chem Biol Interact 293, 70-76.

41. Caudill MA (2010) Pre- and postnatal health: evidence of increased choline needs. J Am Diet Assoc 110, 1198-1206.

42. Yan J, Jiang X, West AA, et al. (2013) Pregnancy alters choline dynamics: results of a randomized trial using stable isotope methodology in pregnant and nonpregnant women. Am J Clin Nutr 98, 1459-1467.

43. Ng SK, Olog A, Spinks AB, et al. (2010) Risk factors and obstetric complications of large for gestational age births with adjustments for community effects: results from a new cohort study. BMC Public Health 10, 460-469.

44. Bin P, Azad MAK, Liu G, et al. (2018) Effects of different levels of methionine on sow health and plasma metabolomics during late gestation. Food Funct 9, 4979-4988.

45. Di Cianni G, Miccoli R, Volpe L, et al. (2005) Maternal triglyceride levels and newborn weight in pregnant women with normal glucose tolerance. Diabet Med 22, 21-25.

46. Mossayebi E, Arab Z, Rahmaniyan M, et al. (2014) Prediction of neonates' macrosomia with maternal lipid profile of healthy mothers. Pediatr Neonatol 55, 28-34.

47. Alder J, Fink N, Bitzer J, et al. (2007) Depression and anxiety during pregnancy: a risk factor for obstetric, fetal and neonatal outcome? A critical review of the literature. J Matern Fetal Neonatal Med 20, 189-209. 